\title{
Effectiveness of concurrent care to improve pediatric and family outcomes at the end of life: An analytic codebook
}

\author{
Radion Svynarenko \\ University of Tennessee, Knoxville \\ Theresa L. Profant \\ University of Tennessee, Knoxville \\ Lisa C. Lindley \\ University of Tennessee, Knoxville
}

Follow this and additional works at: https://trace.tennessee.edu/utk_nurspubs

Part of the Pediatric Nursing Commons

\section{Recommended Citation}

Svynarenko, Radion; Profant, Theresa L.; and Lindley, Lisa C., "Effectiveness of concurrent care to improve pediatric and family outcomes at the end of life: An analytic codebook" (2022). Faculty Publications and Other Works -- Nursing.

https://trace.tennessee.edu/utk_nurspubs/164

This Publication is brought to you for free and open access by the Nursing at TRACE: Tennessee Research and Creative Exchange. It has been accepted for inclusion in Faculty Publications and Other Works -- Nursing by an authorized administrator of TRACE: Tennessee Research and Creative Exchange. For more information, please contact trace@utk.edu. 
Effectiveness of concurrent care to improve pediatric and family outcomes at the end of life: An analytic codebook

Version 1. January 28, 2022 


\section{Funding:}

This publication was made possible by Grant Number R01NR017848 (PI: Lindley) from the National Institute of Nursing Research and the office of the Director. Its contents are solely the responsibility of the authors and do not necessarily represent the official views of the National Institute of Nursing Research or National Institutes of Health, University of Tennessee, Knoxville, or the College of Nursing.

\section{Recommended citation:}

Svynarenko, R., Profant, T., Lindley, L.C., (2022) Effectiveness of concurrent care to improve pediatric and family outcomes at the end of life: An analytic codebook. DOI: 
Table of Contents

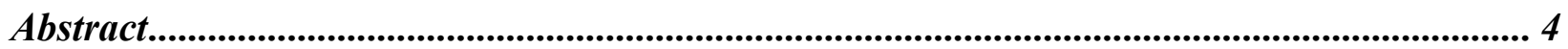

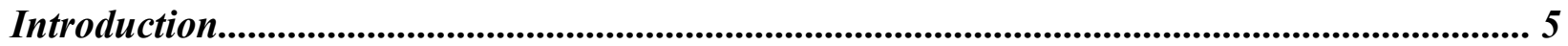

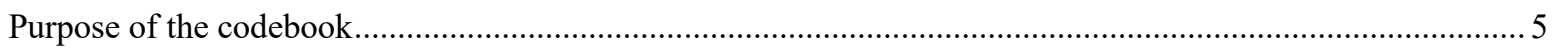

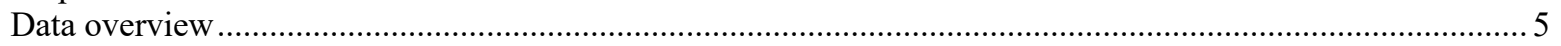

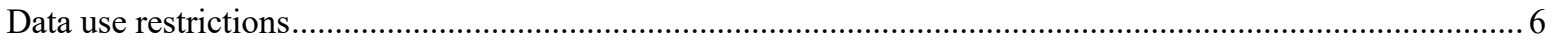

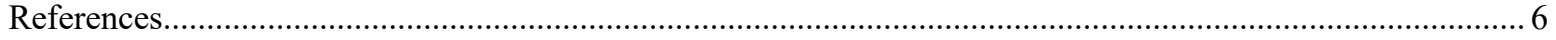

Short List of Variables .............................................................................................................. 8

Frequency Tables and Definitions of Variables ........................................................................... 9

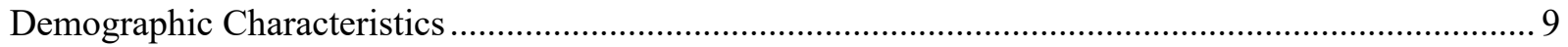

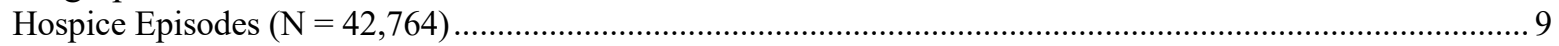

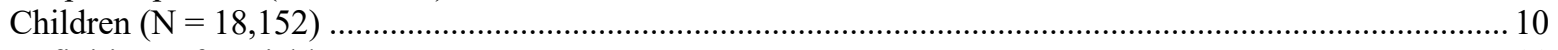

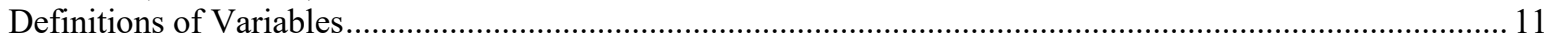

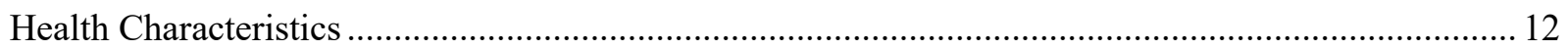

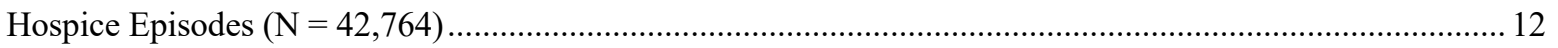

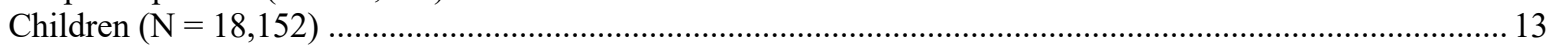

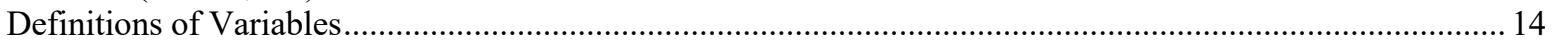

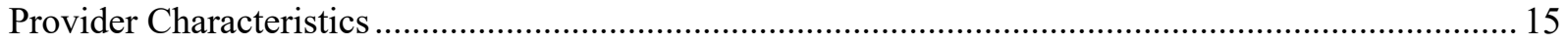

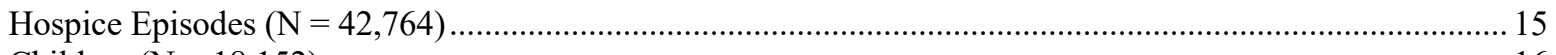

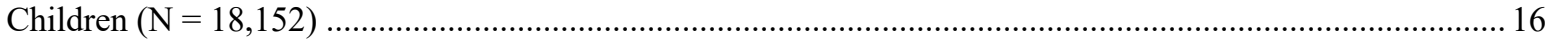

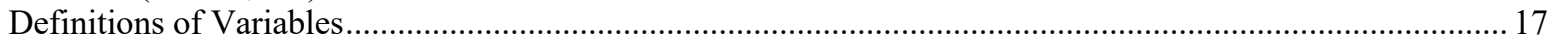

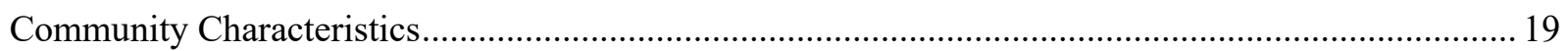

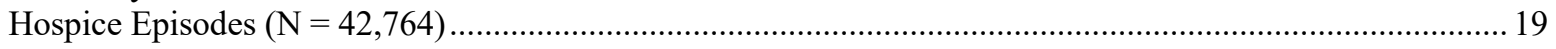

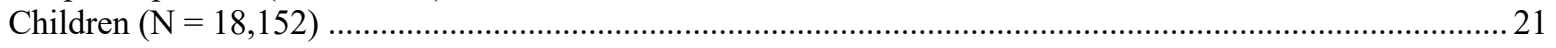

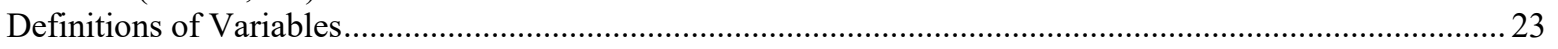

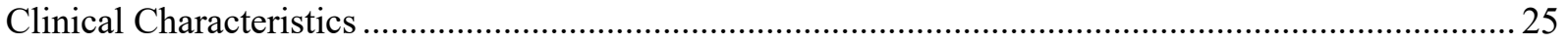

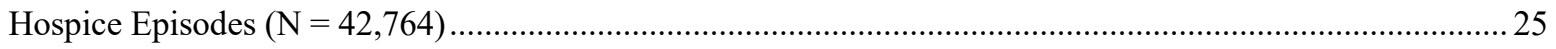

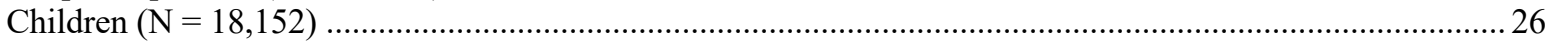

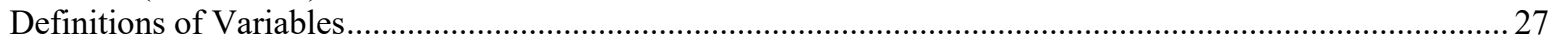

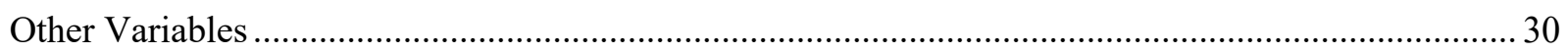

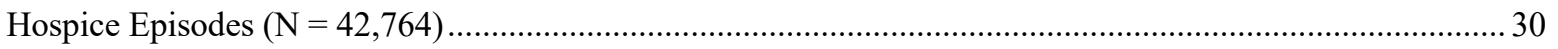

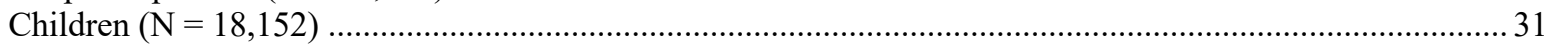

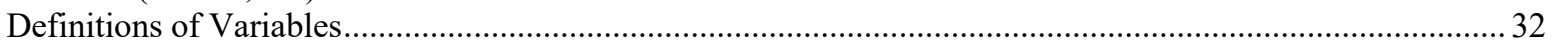

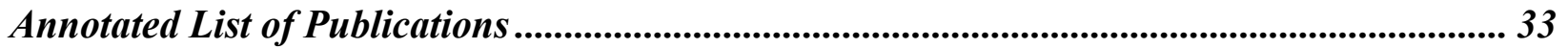

Appendix: Creating Hospice Episodes............................................................................................... 37 


\begin{abstract}
Implementation of the section 2302 of the 2010 Patient Protection and Affordable Care Act (ACA) enabled children enrolled in Medicaid/Children's Health Insurance Program with a prognosis of 6 months to live to use hospice care while continuing treatment for their terminal illness. Although concurrent hospice care became available more than a decade ago, little is known about the socio-demographic and health characteristics of children who received concurrent care; health care services they received while enrolled in concurrent care, their continuity, management, intensity, fragmentation; and the costs of care. The purpose of this study was to answer these questions using national data from the Centers of Medicare and Medicaid Services (CMS), which covered the first three years of ACA - from January 1, 2011, to December 31, 2013.The database included records of 18,152 children younger than the age of 20, who were enrolled in Medicaid hospice care in the sampling time frame. Children in the database also had a total number of 42,764 hospice episodes. Observations were excluded if the date of birth or death was missing or participants were older than 21 years. To create this database CMS data were merged with three other complementary databases: the National Death Index (NDI) that provided information on death certificates of children; the U.S. Census Bureau American Community Survey that provided information on characteristics of communities where children resided; CMS Hospice Provider of Services files and CMS Hospice Utilization and Payment files were used for data on hospice providers, and with a database of rural areas created by the Health Resources and Services Administration (HRSA). In total, 130 variables were created, measuring demographics and health characteristics of children, characteristics of health providers, community characteristics, clinical characteristics, costs of care, and other variables.
\end{abstract} Keywords: concurrent hospice care, end-of-life care, hospice care, Medicaid, pediatric. 


\section{Introduction}

\section{Purpose of the codebook}

The codebook includes documentation for the research project Effectiveness of Concurrent Care to Improve Pediatric and Family Outcomes. This research project was sponsored by the National Institute of Nursing Research and Office of the Director, Grant Number R01NR017848 (PI: Lindley). Additional information about the project can be found online at www.pedeolcare.utk.edu.

This codebook was designed for researchers and analysts interested in using Medicaid data to investigate the utilization of hospice services by pediatric patients. Publications prepared using these variables are available free of charge at PubMed.

\section{Data overview}

Medicaid is an entitlement program that provides health care coverage to low-income families and is the most extensive health insurance program for children younger than $21 .{ }^{1}$ All information about Medicaid enrollees and health services covered by the program are managed by the Medicaid Statistical Information System (MSIS). ${ }^{1}$ It includes data on enrollees from all 50 states and the District of Columbia but excludes those residing in Puerto Rico or other U.S. territories. ${ }^{2}$ Since MSIS was designed for administrative purposes and not research, the Medicaid Analytic Extract (MAX) data system was created as a research tool to provide information on Medicaid enrollment, service utilization, and expenditure at the enrollee level.

The quality of the MAX data is maintained by the Centers for Medicare \& Medicaid Services (CMS). Although each state conducts data quality checks of Medicaid data before submission to CMS, CMS contracts with Mathematica Policy Research to review state data against tolerance criteria and assist states in making corrections, as necessary. Reports have found no evidence of incomplete data or gross miscoding errors. Medicaid managed-care encounter data has also been assessed for completeness and accuracy, and its quality guided the inclusion of states in our study.

For this study, the following CMS Medicaid data files were used:

- MAX Personal Summary Files (PS) combined demographic characteristics with eligibility information, summary claims, and payment data in one record per enrollee.

- MAX Other Therapy Files (OT) were used for diagnosis and procedure information. These files provided claims data on hospice care, along with the International Classification of Diseases ( $9^{\text {th }}$ version ICD-9) and Current Procedural Terminology (CPT) codes.

- MAX Inpatient Files (IP) were included for hospitalization data on service dates and procedure codes

- MAX Prescription Drug (RX) files contained information on prescription fill date and national drug codes, grouped following the Medicaid RX or Red Book classification.

This codebook covers MAX versions from 2011 to 2013. This time frame was selected because it represents the first three full years of implementing the Affordable Care Act and provision 2302 that allowed pediatric Medicaid enrollees to enroll in concurrent hospice care. The major 
limitation of this codebook is that it may be incompatible with CMS databases created after 2016 because starting that year CMS fully transitioned to T-MSIS Analytic Files (TAF), which has a data structure different from MAX.

Other data sources used for the study were:

- National Death Index (NDI) is a database maintained by the Centers for Disease Control and Prevention's National Center for Health Statistics. ${ }^{3}$ NDI is derived from information on death certificates and is updated annually. The database used in this study contained the date of death and causes of death for all US residents from 2011 to 2013. The database used in this study contained the dates and causes of death for all US residents from 2011 to 2013. It was manually linked to the MAX files using the social security numbers of pediatric patients.

- Publicly available U.S. Census Bureau American Community Survey provided information on community income and education. ${ }^{4}$ These data were manually linked to the Medicaid files using Federal Information Processing Standards (FIPS) code. Database from 2010 was used because it included data from the latest census.

- Publicly available CMS Hospice Provider of Services files ${ }^{5}$ and CMS Hospice Utilization and Payment files for $2013^{6}$ were used for information on hospice providers. These data were manually linked by the National Provider Identifier (NPI). Database from 2013 was used because it contained the largest number of hospices.

- A publicly available list of rural areas created by the Health Resources and Services Administration (HRSA) was used to identify rural communities. ${ }^{7}$ The database was merged with the MAX files using FIPS codes.

\section{Data use restrictions}

Medicaid data for this study contained protected health information (PHI) and personally identifiable information (PII) protected under the HIPAA Privacy Rule. The data files are NOT available for research activities because of the Data Use Agreement (DUA) between CMS and the research institution, the University of Tennessee, Knoxville.

\section{References}

1. Kuo DZ, Hall M, Agrawal R, et al. Comparison of health care spending and utilization among children with Medicaid insurance. Pediatrics. 2015;136(6):e1521-1529. doi:10.1542/peds.2015-0871

2. Borck R, Ruttner L, Byrd V, Wagnerman K. Medicaid Analytic Extract 2010 Chartbook. Mathematica Policy Research.Centers for Medicare \& Medicaid services; 2010. Published 2010. Accessed October 21, 2021. https://www.cms.gov/Research-Statistics-Data-andSystems/Computer-Data-and-Systems/MedicaidDataSourcesGenInfo/MAX_Chartbooks

3. National Death Index. Centers for Disease Control and Prevention. Published 2013. Updated February 8, 2021. Accessed October 28, 2021. https://www.cdc.gov/nchs/ndi/index.htm

4. American Community Survey. U.S. Census Bureau. Updated October 12, 2021. Accessed October 28, 2021. https://www.census.gov/programs-surveys/acs 
5. Hospice Providers of Services. Centers for Medicare and Medicaid Services. Published 2013. Updated February 11, 2020. Accessed October 28, 2021. https://www.cms.gov/ResearchStatistics-Data-and-Systems/Downloadable-Public-Use-Files/Provider-of-Services/POS2013

6. Medicare Provider Utilization and Payment Data. Centers for Medicare and Medicaid Services. Updated December 3, 2020. Accessed October 28, 2021. https:/www.cms.gov/Research-Statistics-Data-and-Systems/Statistics-Trends-andReports/Medicare-Provider-Charge-Data

7. Medically Underserved Areas. Health Resources \& Services Administration. Updated October 2021. Accessed October 28, 2021. https://data.hrsa.gov/tools/shortage-area/muafind.

8. Svynarenko R, Beebe LH, Lindley LC. Identifying Patterns of Pediatric Mental and Behavioral Health at End of Life: A National Study. Journal of Hospice \& Palliative Nursing. 2021;Publish Ahead of Print. doi:10.1097/NJH.0000000000000800

9. Feudtner C, Feinstein JA, Zhong W, Hall M, Dai D. Pediatric complex chronic conditions classification system version 2: Updated for ICD-10 and complex medical technology dependence and transplantation. BMC Pediatr. 2014;14(1):199. doi:10.1186/1471-2431-14199

10. STATA. Working with dates and times. Accessed January13, 2022. https://www.stata.com/manuals13/u24.pdf 


\section{Short List of Variables}

\begin{tabular}{|c|c|c|c|}
\hline Characteristics & Variable names & Characteristics & Variable names \\
\hline Demographics & & 46. Region [cat.] & region \\
\hline 0. Age in years [con.] & age_yrs & 47. Northeast [bin.] & region_neast \\
\hline 1. Age groups [cat.] & age groups & 48. Midwest [bin.] & region_mwest \\
\hline 2. 0 to 1 years [bin.] & age $0 \_1$ & 49. South [bin.] & region_south \\
\hline 3. 1 to 5 years [bin.] & age1_5 & 50. West [bin.] & region_west \\
\hline 4. 6 to 14 years [bin.] & age6_14 & 51. Appalachia [bin.] & region_app \\
\hline 5. 15 to 20 years [bin.] & age15_20 & 52. Southern Appalachia [bin.] & region_sapp \\
\hline 6. Gender [bin.] & gender & 53. Rurality [bin.] & ru hrsa 2 \\
\hline Race & & 54. U.S. States [str.] & state_cd \\
\hline 7. White [bin.] & white & 55. to 100. U.S.States [bin.] & $\mathrm{AK}, \overline{\mathrm{AL}}, \mathrm{AR}, \mathrm{CA}, \mathrm{CO}, \mathrm{CT}$, \\
\hline 8. Black [bin.] & black & & DC, DE, FL, GA, HI, IA, ID, \\
\hline 9. Ethnicity [bin.] & hispanic & & IL, IN, KS, KY, LA, MA, \\
\hline Health Characteristics & & & MD, ME, MI, MN, MO, MS, \\
\hline 10. Neurologic and neurom. [bin.] & ccc_neuro & & $\mathrm{MT}, \mathrm{NC}, \mathrm{ND}, \mathrm{NJ}, \mathrm{NM}, \mathrm{NV}$ \\
\hline 11. Cardiovascular [bin.] & ccc_cardiac & & $\mathrm{NY}, \mathrm{OH}, \mathrm{OK}, \mathrm{OR}, \mathrm{PA}, \mathrm{SC}$ \\
\hline 12. Respiratory [bin.] & ccc_resp & & $\begin{array}{l}\mathrm{SD}, \mathrm{TN}, \mathrm{TX}, \mathrm{UT}, \mathrm{VA}, \mathrm{WI}, \\
\mathrm{WV} \text {, and WY. }\end{array}$ \\
\hline 13. Renal and urologic [bin.] & ccc_renal & Clinical characteristics & \\
\hline 14. Gastrointestinal [bin.] & ccc_gastro & 101. Hospice LOS [con.] & total los \\
\hline 15. Hematologic and immun. [bin.] & ccc_hemat & 102. Medical Team Size [con.] & coord_phy \\
\hline 16. Metabolic [bin.] & ccc_metabolic & 103. Hospice Live Discharge [con.] & fhospice_live_disch_episode \\
\hline 17. Congenital and Genetic [bin.] & ccc_congenital & 104. Symptom management [bin.] & sym_mgt_icd $\overline{9}$ \\
\hline 18. Malignancy [bin.] & ccc_cancer & 105. 1day Hospice [bin.] & care_intensity_1day \\
\hline 19. Premature and neonatal [bin.] & ccc- neonatal & 106. Hospice Transition ER [bin.] & transition er \\
\hline 20. Miscellaneous conditions [bin.] & ccc_mis & 107. Primary Care Visits [bin.] & physician_use \\
\hline 21. Mental health [bin.] & mental_health & 108. Hospice Transition Inpat [bin.] & transition_inpt \\
\hline 22. Technology dependent [bin.] & function_status & 109. Coordination Insurance [bin.] & coord ins ${ }^{-}$ \\
\hline 23. Medical complexity [bin.] & med_complex & 110. \# of episodes per child [con.] & ftotal_epi \\
\hline 24. Any medical complexity [bin.] & med_complex_any & 111. Concurrent care (rx) [bin.] & concurrent_care_rx \\
\hline 25. Increase medical compl. [bin.] & increase_med_complex & Costs & \\
\hline 26. Health condition [bin.] & $\operatorname{ccc}-1-10$ & 112. Total costs [con.] & icosts_tot \\
\hline 27. Health severity [bin.] & mecc & 113. Fee-for-services [con.] & icosts_tot_ffs \\
\hline Provider Characteristics & & 114. Premium payment [con.] & icosts_tot_prem \\
\hline 28. Live discharge [con.] & iTotal_Live_Dis & 115. Inpatient [con.] & icosts_tot_ip \\
\hline 29. Short stay pts [con.] & iHbene_7orless_hospcaredays & 116. Outpatient [con.] & icosts_tot_ot \\
\hline 30. Medicaid pts [con.] & iMedicĒlig_hospbene & 117. Prescription drugs [con.] & icosts_tot_rx \\
\hline 31. Hospice Size [cat.] & Hospice_size & 118. Labs [con.] & icosts_tot_lab \\
\hline 32. Less than 5 [bin.] & hospice_mini & 119. Transportation [con.] & icosts_tot_transport \\
\hline 33. Micro 5-9 [bin.] & hospice_micro & 120. Therapies [con.] & icosts_tot_therapies \\
\hline 34. Small 10-49 [bin.] & hospice_small & 121. Hospice benefits [con.] & icosts_tot_hb \\
\hline 35. Medium and large, $>50$ [bin.] & hospice_large & 122. Registered nurse visits [con.] & icosts_tot_RN \\
\hline 36. Hospice Ownership [cat.] & hospice_ownership & 123. Durable med. equip. [con.] & icosts_tot_dme \\
\hline 37. Hospice Age [con.] & hospice_age & Other Variables & \\
\hline 38. Skilled RN visits [con.] & ihospice_rnvisits & 124. Medicaid waiver [bin.] & waiver \\
\hline 39. Pediatric programs [bin.] & pediatric_prgrm & 125. Palliative policy [bin.] & ped_pall_policy \\
\hline 40. Care coordination [bin.] & care coordination & 126. Hospice's postal ZIP code [str.] & ziphosp \\
\hline Community Characteristics & & 127. Patient's postal ZIP code [str.] & ZIP \\
\hline Education & & Years & \\
\hline 41. No high school diploma [con.] & edf_nohs_pc & 128. 2011 [bin.] & y2011 \\
\hline 42. High school diploma [con.] & edf_hs_ps & 129. 2012 [bin.] & y2012 \\
\hline 43. Some college [con.] & edf_college_pc & 130. 2013 [bin.] & y2013 \\
\hline 44. College degree [con.] & edf_collegedegree_pc & & \\
\hline 45. Income less than 50,000 [bin.] & iincome less50k bin & & \\
\hline
\end{tabular}

Note. [con.] indicates a continuous variable, [bin.] - binary, [cat.] - categorical, and [str.]- a string variable with text. 


\section{Frequency Tables and Definitions of Variables Demographic Characteristics}

\begin{tabular}{|c|c|c|c|c|c|c|c|}
\hline$\#$ & Characteristic & Variable & $\mathbf{n}$ & $\%$ & M(SD) & Min. & Max. \\
\hline 0 . & Age in years & age_yrs & - & - & $7.45(6.3)$ & 0 & 20 \\
\hline 1. & Age groups & age_groups & & & & 0 & 3 \\
\hline 2. & Age 0 to 1 years & age $0 \_1$ & 5,536 & $12.95 \%$ & & & \\
\hline 3. & Age 1 to 5 years & age1_5 & 14,568 & $34.07 \%$ & & & \\
\hline 4. & Age 6 to 14 years & age6_14 & 14,437 & $33.76 \%$ & & & \\
\hline 5. & Age 15 to 20 years & age15_20 & 8,223 & $19.23 \%$ & & & \\
\hline \multirow[t]{4}{*}{6.} & Gender & gender & & & & 0 & 1 \\
\hline & 0. Male & & 22,248 & $52.03 \%$ & & & \\
\hline & 1. Female & & 20,516 & $47.97 \%$ & & & \\
\hline & Race & & & & & & \\
\hline \multirow[t]{3}{*}{7.} & White & white & & & & 0 & 1 \\
\hline & 0.non-White & & 22,697 & $53.08 \%$ & & & \\
\hline & 1.White & & 20,067 & $46.92 \%$ & & & \\
\hline \multirow[t]{3}{*}{8.} & Black & black & & & & 0 & 1 \\
\hline & 1. Black & & 9,397 & $21.97 \%$ & & & \\
\hline & 0. non-Black & & 33,367 & 78.03 & & & \\
\hline \multirow[t]{3}{*}{9.} & Ethnicity & hispanic & & & & 0 & 1 \\
\hline & 1. Hispanic & & 12,074 & $28.23 \%$ & & & \\
\hline & 0. non-Hispanic & & 30,960 & $71.77 \%$ & & & \\
\hline
\end{tabular}


Children $(\mathrm{N}=18,152)$

\begin{tabular}{|c|c|c|c|c|c|c|c|}
\hline$\#$ & Characteristic & Variable & n & $\%$ & M(SD) & Min. & Max. \\
\hline 0. & Age in years & age_yrs & & & $7.08(6.3)$ & 0 & 20 \\
\hline 1. & Age groups & age_groups & & & & 0 & 3 \\
\hline 2. & Age 0 to 1 years & age $0 \_1$ & 2,776 & $15.29 \%$ & & & \\
\hline 3. & Age 1 to 5 years & age1_5 & 6,552 & $36.10 \%$ & & & \\
\hline 4. & Age 6 to 14 years & age6_14 & 5,575 & $30.71 \%$ & & & \\
\hline 5. & Age 15 to 20 years & age15_20 & 3,249 & $17.90 \%$ & & & \\
\hline 6. & Gender & gender & & & & 0 & 1 \\
\hline & 0. Male & & 9,297 & $51.22 \%$ & & & \\
\hline & 1. Female & & 8,855 & $48.78 \%$ & & & \\
\hline 7. & White & white & & & & 0 & 1 \\
\hline & 0.non-White & & 8,385 & $46.19 \%$ & & & \\
\hline & 1.White & & 9,767 & $53.81 \%$ & & & \\
\hline 8. & Black & black & & & & 0 & 1 \\
\hline \multirow{5}{*}{9.} & 1. Black & & 4,938 & $27.20 \%$ & & & \\
\hline & 0. non-Black & & 13,226 & $72.86 \%$ & & & \\
\hline & Ethnicity & hispanic & & & & 0 & 1 \\
\hline & 1. Hispanic & & 4,001 & $22.04 \%$ & & & \\
\hline & 0. non-Hispanic & & 14,151 & $77.96 \%$ & & & \\
\hline
\end{tabular}




\section{Definitions of Variables}

Variables 0 to 9. These variables indicate sociodemographic characteristics of hospice patients. They were created using MAX PS files.

0. Age in years variable (age_yrs) is calculated by subtracting birthdate (s_el_dob_y) from the year of data collection (yr_num).

1. Age groups (age_groups) is created by recording existing age groups (el_age_grp_cd) into four age categories: 0.) 0 to 1 years old, 1.) 1 to 5 years old, 2.) 6 to 14 years old, 3.) 15 to 20 years old. These categories were saved into separate dummies:

2. Age 0 to 1 (age $\left.0 \_1\right)$,

3. Age 1 to 5 years (age1_5),

4. Age 6 to 14 years (age6_14),

5. Age 15 to 20 years (age 15_20).

6. Gender variable (gender) is created by recoding the existing variable (el_sex_cd) into a new with two categories: male and female

7. White race (white) is created by recoding the dummy variable (race_code_1).

8. Black race (black) is created by recoding the dummy variable (race_code_2).

9. Hispanic ethnicity (hispanic) is created by recoding a categorical variable (ethnicity_code) with value $=1$. 


\section{Health Characteristics}

\begin{tabular}{|c|c|c|c|c|c|c|c|}
\hline \multicolumn{8}{|c|}{ Hospice Episodes $(N=42,764)$} \\
\hline \# & Characteristic & Variable name & $\mathbf{n}$ & $\%$ & M(SD) & Min. & Max. \\
\hline \multirow[t]{2}{*}{10.} & \multicolumn{2}{|l|}{ Neurologic and } & & & & \multirow[t]{2}{*}{0} & \multirow[t]{2}{*}{1} \\
\hline & neuromuscular & ccc neuro & 14,142 & $33.07 \%$ & & & \\
\hline 11. & Cardiovascular & ccc_cardiac & 13,698 & $32.03 \%$ & & 0 & 1 \\
\hline 12. & Respiratory & ccc_resp & 10,104 & $23.63 \%$ & & 0 & 1 \\
\hline 13. & Renal and urologic & ccc renal & 3,921 & $9.17 \%$ & & 0 & 1 \\
\hline 14. & Gastrointestinal & ccc_gastro & 13,050 & $30.52 \%$ & & 0 & 1 \\
\hline 15. & Hematologic and immun. & ccc hemat & 5,006 & $11.71 \%$ & & 0 & 1 \\
\hline 16. & Metabolic & ccc_metabolic & 7,082 & $16.56 \%$ & & 0 & 1 \\
\hline 17. & Other congenital and Genetic & ccc_congenital & 9,044 & $21.15 \%$ & & 0 & 1 \\
\hline 18. & Malignancy & ccc_cancer & 6,009 & $14.05 \%$ & & 0 & 1 \\
\hline 19. & Premature and neonatal & ccc_neonatal & 4,225 & $9.88 \%$ & & 0 & 1 \\
\hline 20. & Miscellaneous & ccc_mis & 18,457 & $43.20 \%$ & & 0 & 1 \\
\hline 21. & Mental health & mental_all & 20,257 & $47.37 \%$ & & 0 & 1 \\
\hline 22. & Technology dependence & function_status & 16,417 & $38.39 \%$ & & 0 & 1 \\
\hline 23. & Med complexity & med complex & 11,745 & $27.46 \%$ & & 0 & 1 \\
\hline 24. & Med complexity, any & med_complex_any & 31,022 & $72.54 \%$ & & 0 & 1 \\
\hline 25 . & Increase medical complexity & increase_med_compl & - & - & $1.23(1.28)$ & 0 & 3 \\
\hline 26. & Health condition & $\mathrm{ccc}$ & 27,465 & $64.22 \%$ & & 0 & 1 \\
\hline 27. & Health severity & mec & 19,464 & $45.51 \%$ & & 0 & 1 \\
\hline
\end{tabular}


Children $(\mathrm{N}=18,152)$

\begin{tabular}{|c|c|c|c|c|c|c|c|}
\hline$\#$ & Characteristic & Variable name & $\mathbf{n}$ & $\%$ & M(SD) & Min. & Max. \\
\hline \multirow[t]{2}{*}{10.} & Neurologic and & & & & & 0 & 1 \\
\hline & neuromuscular & ccc_neuro & 13053 & $16.82 \%$ & & & \\
\hline 11. & Cardiovascular & ccc_cardiac & 3694 & $20.35 \%$ & & 0 & 1 \\
\hline 12. & Respiratory & ccc_resp & 2024 & $11.15 \%$ & & 0 & 1 \\
\hline 13. & Renal and urologic & ccc renal & 893 & $4.92 \%$ & & 0 & 1 \\
\hline 14. & Gastrointestinal & ccc_gastro & 2695 & $14.85 \%$ & & 0 & 1 \\
\hline 15. & Hematologic and immun. & ccc_hemat & 1312 & $7.23 \%$ & & 0 & 1 \\
\hline 16. & Metabolic & ccc_metabolic & 1956 & $10.78 \%$ & & 0 & 1 \\
\hline 17. & Congenital and genetic & ccc_congenital & 1954 & $10.76 \%$ & & 0 & 1 \\
\hline 18. & Malignancy & ccc_cancer & 1690 & $9.31 \%$ & & 0 & 1 \\
\hline 19. & Premature and neonatal & ccc_neonatal & 629 & $3.47 \%$ & & 0 & 1 \\
\hline 20. & Miscellaneous & ccc_mis & 4974 & $27.40 \%$ & & 0 & 1 \\
\hline 21. & Mental health & mental_all & 6,195 & $34.13 \%$ & & 0 & 1 \\
\hline 22. & Technology dependence & function_status & 4,160 & $22.92 \%$ & & 0 & 1 \\
\hline 23. & Med complexity & med_complex & 2,357 & $12.98 \%$ & & 0 & 1 \\
\hline 24. & Med complexity, any & med_complex_any & 10,856 & $59.81 \%$ & & 0 & 1 \\
\hline 25. & Increase medical complexity & increase_med_compl & - & - & $.74(1.10)$ & 0 & 3 \\
\hline 26. & Health condition & $\operatorname{ccc}$ & 8784 & $48.39 \%$ & & 0 & 1 \\
\hline 27. & Health severity & mec & 5088 & $28.03 \%$ & & 0 & 1 \\
\hline
\end{tabular}




\section{Definitions of Variables}

Variables 10 to 20. All health characteristics of patients were extracted from MAX IP files which retained ICD-9 codes for each medical condition (variables diag_cd_*). Using this data and definitions of complex chronic conditions variables published by Feudtner et al., (2014), our team created 11 dummy variables ( $\mathrm{ccc}_{-}^{*}$ ) for each primary complex chronic condition including:

10. Neurologic and neuromuscular (ccc_neuro),

11. Cardiovascular (ccc_cardiac),

12. Respiratory (ccc_resp),

13. Renal and urologic (ccc_renal),

14. Gastrointestinal (ccc_gastro),

15. Hematologic or immunologic (ccc_hemat),

16. Metabolic (ccc_metabolic),

17. Congenital or genetic defect (ccc_congenital),

18. Malignancy (ccc_cancer),

19. Premature and neonatal (ccc_neonatal),

20. Miscellaneous (ccc_misc).

Variables 21 to 27. Our team also created seven additional health variables including:

21. Mental health (mental_all) indicates having any mental and behavioral health conditions: anxiety (ICD-9-CM: 300, 308), neurodevelopment disorders (ICD-9-CM: 299, 314), psychotic disorders (ICD-9- CM: 293-295, 297, 298, 316), substance misuse (ICD-9-CM: 291-292, 303-305), cognitive (ICD-9-CM: 290, 310, 315, 317-319), mood disorders (ICD-9CM: 300.4, 296.1-296.9, 311), behavioral disorders (ICD-9-CM: 312-313), and other mental health conditions (ICD-9-CM: 301-302, 306-307). ${ }^{8}$

22. Technology dependency (function_status) is defined according to Feudtner's definition. ${ }^{9}$

23. Medical complexity (med_complex) indicates having all four medical conditions, including ccc, mccc, function_status, and mental health

24. Any Medical complexity (med_complex_any) indicates having any one of the four complex conditions (including ccc, mccc, function_status, and mental health).

25. Increased medical complexity (increase_med_compl) indicates the number of complex conditions (including ccc, mccc, function_status, and mental health).

26. Health conditions ( $\mathrm{ccc}$ ) indicates having any of the 11 primary complex chronic conditions.

27. Health severity (mccc) indicates having two or more of the 11 primary complex chronic conditions. 


\section{Provider Characteristics}

Hospice Episodes $(\mathrm{N}=42,764)$

\begin{tabular}{|c|c|c|c|c|c|c|c|}
\hline$\#$ & Characteristics & Variable name & n & $\%$ & M(SD) & Min. & Max. \\
\hline 28. & Live discharge patients & iTotal_Live_Dis & - & - & $114.8(130.34)$ & & 2,217 \\
\hline 29. & Short-stay patients & iHbene 7orless hospcaredays & - & - & $345.92(405.59)$ & & 5,383 \\
\hline 30. & Medicaid patients & iMedicĒlig_hospbene & - & - & $329.19(528.49)$ & 11 & 6,744 \\
\hline 31. & Hospice size, employees & Hospice_size & & & & 0 & \\
\hline 32. & Less than 5 & hospice_mini & 7,527 & $17.60 \%$ & & & \\
\hline 33. & Micro, 5 to 9 & hospice_micro & 6,303 & $14.74 \%$ & & & \\
\hline 34. & Small, 20 to 49 & hospice_small & 11,262 & $26.34 \%$ & & & \\
\hline 35 . & Medium to large, $50+$ & hospice_large & 17,672 & $41.32 \%$ & & & \\
\hline \multirow[t]{3}{*}{36.} & Hospice ownership & hospice_ownership & & & & 0 & \\
\hline & 1.Non-profit/ Government & & 16,605 & $38.83 \%$ & & & \\
\hline & 0.For profit & & 26,159 & $61.17 \%$ & & & \\
\hline 37. & Hospice age, years & ce_age & & & $18.30(10.04)$ & .70 & 30.2 \\
\hline 38. & Hospice RN visits & ihospice_rnvisits & & & $.34(.12)$ & 0 & \\
\hline 39. & Pediatric program & pediatric_prgrm & 15,037 & $35.16 \%$ & & 0 & \\
\hline 40. & Care coordination & care coordination & 42,744 & $99.95 \%$ & & & \\
\hline
\end{tabular}


Children $(\mathrm{N}=18,152)$

\begin{tabular}{|c|c|c|c|c|c|c|c|}
\hline \# & Characteristics & Variable name & $\mathbf{n}$ & $\%$ & M/SD & Min. & Max. \\
\hline 28. & Live discharge patients & iTotal Live Dis & - & - & $103.95 / 128.88$ & 11 & 2,217 \\
\hline 29. & Short-stay patients & iHbene_7orless_hospcaredays & - & - & $310.52 / 393.43$ & 0 & 5,383 \\
\hline 30. & Medicaid patients & iMedicElig_hospbene & - & - & $295.23 / 464.80$ & 11 & 6,744 \\
\hline 31. & Hospice size, employees & Hospice_size & - & - & & & \\
\hline 32. & Less than 5 & hospice_mini & 4,019 & $22.14 \%$ & & 0 & 1 \\
\hline 33. & Micro, 5 to 9 & hospice_micro & 3,986 & $21.63 \%$ & & 0 & 1 \\
\hline 34. & Small, 20 to 49 & hospice_small & 3,710 & $21.96 \%$ & & 0 & 1 \\
\hline 35. & Medium to large, $50+$ & hospice_large & 6,437 & $35.46 \%$ & & 0 & 1 \\
\hline \multirow[t]{3}{*}{36.} & Hospice ownership & hospice_ownership & & & & & \\
\hline & 1.Non-profit/ Government & & 7,011 & $38.62 \%$ & & 0 & 1 \\
\hline & 0.For profit & & 11,141 & $61.38 \%$ & & 0 & 1 \\
\hline 37. & Hospice age, years & hospice_age & & & $16.56 / 9.82$ & .70 & 30.2 \\
\hline 38. & Hospice RN visits & ihospice_rnvisits & & & $.34 / .13$ & 0 & 2.7 \\
\hline 39. & Pediatric program & pediatric_prgrm & 6,090 & $33.55 \%$ & & 0 & 1 \\
\hline 40. & Care coordination & care_coordination & 18,148 & $99.98 \%$ & & 0 & 1 \\
\hline
\end{tabular}




\section{Definitions of Variables}

Provider characteristics were extracted from the publicly available CMS Hospice Provider of Services files (POS) and CMS Hospice Utilization and Payment files (PUF). ${ }^{5}$ The following variables were created:

28. Live discharge patients (iTotal_Live_Dis) indicates the number of distinct patients with live discharges from hospice care. "A hospice beneficiary was considered to have a live discharge if the hospice beneficiary did not die in hospice care and was not receiving hospice care. Includes live discharges for any reason including revocation" (PUF manual, p.7). It was created using (iTotal_Live_Dis) from the PUF file.

29. Short-stay patients (iHbene_7orless_hospcaredays) indicates the number of patients with 7 or fewer hospice care days. Excludes hospice beneficiaries whose hospice care continued from a previous calendar year or into the next calendar year (PUF manual, p.7). It was created using (Total_Live_Dis) from the PUF file.

30. Medicaid patients (iMedicElig_hospbene) indicates the number patients eligible for Medicaid for at least one month and receiving at least one day of hospice care in the calendar year (PUF manual, p.7). It was created using (MedicElig_hospbene) from the PUF file.

31. Hospice size (hospice_size) indicates the number of employees working in the hospice. It was created using the Employee Count variable (emplee_cnt) from the POS file. Four subcategories of hospices were identified: 1.) Mini hospice with less than 7 employees; 2.) Micro hospice with 5 to 9 emoployees; 3.) Small hospice with 20 to 49 employees, and 0.) Medium to large hospice with 50 or more employees. These categories were also were saved as respective dummies:

32. Mini hospice with less than 7 employees (hospice_mini).

33. Micro hospice with 5 to 9 employees (hospice_micro).

34. Small hospics with 20 to 49 employees (hospice_small).

35. Medium to large hospices with 50 employees or more (hospice_large).

36. The hospice ownership (hospice ownership) indicates two types of hospice ownership: 1.) Non-profit or Governmental, and 0.) For-profit. It was was created using the Ownership type of the provider variable (gnrl_cntl_type_cd) from the POS file.

37. Hospice age in years (hospice_age) indicates the age of the hospice. It was calculated by substracting the date when the provider was first approved to provide Medicare and/or Medicaid service variable (orgnl_prtcptn_dt) from the last day of 2013 and divided by 365.25. ${ }^{10}$ The (orgnl_prtcptn_dt) variable was extracted from the POS file. 
38. Hospice RN visits (ihospice_rnvisits) indicates the average number of hours per day of skilled nursing hospice care provided. Skilled nursing visits were identified using Revenue Codes 0550, 0551, 0552, and 0559 (PUF manual, p.6). It was created using (hospice_rnvisits) variable from the PUF file.

39. Pediatric program (pediatric_prgrm) indicates whether a hospice provided any pediatric services and was created using publicly available data.

40. Care coordination (care_coordination) indicates any transitional care management (TCM), patient-centered medical home care, or managed care. It was created using variables (enctr_rec_cnt_31;ffs_clm_cnt_31;ffs_pymt_amt_31;ffs_chrg_amt_31; ffs_tp_amt_31; cltc_ffs_pymt_amt_17; cltc_ffs_pymt_amt_37; mc_combo_mo_1 through $12 \overline{2}$; el_pph_pln_mo_cnt_cmcp) from the PS MAX file. 


\section{Community Characteristics}

Hospice Episodes $(N=42,764)$

\begin{tabular}{|c|c|c|c|c|c|c|c|}
\hline \# & Characteristic & Variable name & $\mathbf{n}$ & $\%$ & M(SD) & Min. & Max. \\
\hline & Education & & & & & & \\
\hline 41. & No high school & edf nohs pc & - & & $14.01(4.39)$ & 3.41 & 38.21 \\
\hline 42. & High school & edf hs_ps & - & - & $24.17(9.59)$ & 12.35 & 52.46 \\
\hline 43. & Some college & edf_college_pc & - & - & $24.49(6.88)$ & 14.34 & 45.44 \\
\hline 44. & College degree & edf collegedegree pc & - & & $37.33(14.95)$ & 5.83 & 59.98 \\
\hline 45. & Income less than $\$ 50,000$ & income_less $50 \mathrm{k}$ bin & 11,874 & $27.77 \%$ & & & \\
\hline 46. & Region & region & & & & 0 & 3 \\
\hline 47. & Northeast & region_neast & 22,207 & $51.93 \%$ & & 0 & 1 \\
\hline 48. & Midwest & region_mwest & 6,965 & $16.29 \%$ & & 0 & 1 \\
\hline 49. & South & region_south & 4,260 & $9.96 \%$ & & 0 & 1 \\
\hline 50. & West & region_west & 9,332 & $21.82 \%$ & & 0 & 1 \\
\hline 51. & Appalachia & region_app & 4,350 & $10.17 \%$ & & 0 & 1 \\
\hline 52. & Southern Appalachia & region_sapp & 1,860 & $4.35 \%$ & & 0 & 1 \\
\hline 53. & Rurality & ru_hrsa 2 & & & & 0 & 1 \\
\hline & 1. Urban & & 24,873 & $58.16 \%$ & & & \\
\hline & 0. Rural & & 17,891 & $41.84 \%$ & & & \\
\hline 54. & U.S. States & state_cd & & & & & \\
\hline 55. & & $\mathrm{AK}$ & 2 & $0 \%$ & & 0 & 1 \\
\hline 56. & & $\mathrm{AL}$ & 66 & $0.15 \%$ & & 0 & 1 \\
\hline 57. & & $\mathrm{AR}$ & 125 & $0.29 \%$ & & 0 & 1 \\
\hline 58. & & $\mathrm{CA}$ & 8107 & $18.96 \%$ & & 0 & 1 \\
\hline 59. & & $\mathrm{CO}$ & 219 & $0.51 \%$ & & 0 & 1 \\
\hline 60. & & $\mathrm{CT}$ & 12 & $0.03 \%$ & & 0 & 1 \\
\hline 61. & & $\mathrm{DC}$ & 2 & $0 \%$ & & 0 & 1 \\
\hline 62. & & $\mathrm{DE}$ & 22 & $0.05 \%$ & & 0 & 1 \\
\hline 63. & & FL & 817 & $1.91 \%$ & & 0 & 1 \\
\hline 64. & & GA & 349 & $0.82 \%$ & & 0 & 1 \\
\hline 65. & & HI & 17 & $0.04 \%$ & & 0 & 1 \\
\hline 66. & & IA & 54 & $0.13 \%$ & & 0 & 1 \\
\hline 67. & & ID & 15 & $0.04 \%$ & & 0 & 1 \\
\hline 68. & & IL & 84 & $0.20 \%$ & & 0 & 1 \\
\hline 69. & & IN & 185 & $0.43 \%$ & & 0 & 1 \\
\hline 70. & & $\mathrm{KS}$ & 87 & $0.20 \%$ & & 0 & 1 \\
\hline 71. & & KY & 402 & $0.94 \%$ & & 0 & 1 \\
\hline 72. & & LA & 78 & $0.18 \%$ & & 0 & 1 \\
\hline 73. & & MA & 305 & $0.71 \%$ & & 0 & 1 \\
\hline 74. & & MD & 575 & $1.34 \%$ & & 0 & 1 \\
\hline 75. & & $\mathrm{ME}$ & 2 & $0 \%$ & & 0 & 1 \\
\hline 76. & & MI & 680 & $1.59 \%$ & & 0 & 1 \\
\hline 77. & & $\mathrm{MN}$ & 38 & $0.09 \%$ & & 0 & 1 \\
\hline 78. & & $\mathrm{MO}$ & 77 & $0.18 \%$ & & 0 & 1 \\
\hline 79. & & MS & 130 & $0.30 \%$ & & 0 & 1 \\
\hline 80. & & MT & 13 & $0.03 \%$ & & 0 & 1 \\
\hline
\end{tabular}




\begin{tabular}{llrrrr}
81. & NC & 157 & $0.37 \%$ & 0 & 1 \\
82. & ND & 4 & $0.01 \%$ & 0 & 1 \\
83. & NJ & 14 & $0.03 \%$ & 0 & 1 \\
84. & NM & 598 & $1.4 \%$ & 0 & 1 \\
85. & NV & 39 & $0.09 \%$ & 0 & 1 \\
86. & NY & 19,238 & $44.99 \%$ & 0 & 1 \\
87. & OH & 5,147 & $12.04 \%$ & 0 & 1 \\
88. & OK & 101 & $0.24 \%$ & 0 & 1 \\
89. & OR & 21 & $0.05 \%$ & 0 & 1 \\
90. & PA & 2,632 & $6.15 \%$ & 0 & 1 \\
91. & SC & 79 & $0.18 \%$ & 0 & 1 \\
92. & SD & 16 & $0.04 \%$ & 0 & 1 \\
93. & TN & 424 & $0.99 \%$ & 0 & 1 \\
94. & TX & 680 & $1.59 \%$ & 0 & 1 \\
95. & UT & 300 & $0.70 \%$ & 0 & 1 \\
96. & VA & 141 & $0.33 \%$ & 0 & 1 \\
97. & VT & 4 & $0.01 \%$ & 0 & 1 \\
98. & WI & 593 & $1.39 \%$ & 0 & 1 \\
99. & WV & 112 & $0.26 \%$ & 0 & 1 \\
100. & WY & 1 & $0.00 \%$ & 0 & 1 \\
\hline
\end{tabular}


Children $(\mathrm{N}=18,152)$

\begin{tabular}{|c|c|c|c|c|c|c|c|}
\hline \# & Characteristic & Variable name & n & $\%$ & M(SD) & Min. & Max. \\
\hline & Education & & & & & & \\
\hline 41. & No high school & edf_nohs pc & - & - & $13.39(4.09)$ & 3.41 & 38.21 \\
\hline 42. & High school & edf hs_ps & - & - & $24.93(9.35)$ & 12.35 & 52.46 \\
\hline 43. & Some college & edf_college_pc & - & - & - 23.71(6.87) & 14.34 & 45.44 \\
\hline 44. & College degree & edf_collegedegree_pc & - & & $37.98(15.87)$ & 5.83 & 59.98 \\
\hline 45. & Income, less than & & & & & & \\
\hline & $\$ 50,000$ & income less50k bin & 6,455 & $35.56 \%$ & & & \\
\hline 46. & Region & region & - & & - & 1 & 4 \\
\hline 47. & Northeast & region neast & 4,404 & $24.26 \%$ & & 0 & 1 \\
\hline 48. & Midwest & region_mwest & 10,431 & $57.46 \%$ & & 0 & 1 \\
\hline 49. & South & region_south & 2,132 & $11.75 \%$ & & 0 & 1 \\
\hline 50. & West & region_west & 1,185 & $6.53 \%$ & & 0 & 1 \\
\hline 51. & Appalachia & region_app & 1,788 & $9.85 \%$ & & 0 & 1 \\
\hline 52. & Southern Appalachia & region_sapp & 869 & $4.79 \%$ & & & \\
\hline 53. & Rurality & ru_hrsa2 & & & & & \\
\hline & 1.Urban & & 12,089 & $66.60 \%$ & & & \\
\hline & 0. Rural & & 6,063 & $33.40 \%$ & & & \\
\hline 54. & U.S.States & state_cd & & & & & \\
\hline 55. & & AK & 1 & $0.01 \%$ & & 0 & 1 \\
\hline 56. & & $\mathrm{AL}$ & 43 & $0.24 \%$ & & 0 & 1 \\
\hline 57. & & AR & 59 & $0.33 \%$ & & 0 & 1 \\
\hline 58. & & $\mathrm{CA}$ & 853 & $4.7 \%$ & & 0 & 1 \\
\hline 59. & & $\mathrm{CO}$ & 36 & $0.2 \%$ & & 0 & 1 \\
\hline 60 & & CT & 12 & $0.07 \%$ & & 0 & 1 \\
\hline 61. & & $\mathrm{DC}$ & 2 & $0.01 \%$ & & 0 & 1 \\
\hline 62. & & DE & 13 & $0.07 \%$ & & 0 & 1 \\
\hline 63. & & FL & 241 & $1.33 \%$ & & 0 & 1 \\
\hline 64. & & GA & 204 & $1.12 \%$ & & 0 & 1 \\
\hline 65 & & HI & 10 & $0.06 \%$ & & 0 & 1 \\
\hline 66. & & IA & 36 & $0.2 \%$ & & 0 & 1 \\
\hline 67. & & ID & 13 & $0.07 \%$ & & 0 & 1 \\
\hline 68. & & IL & 73 & $0.4 \%$ & & 0 & 1 \\
\hline 69. & & IN & 158 & $0.87 \%$ & & 0 & 1 \\
\hline 70 & & KS & 58 & $0.32 \%$ & & 0 & 1 \\
\hline 71. & & KY & 86 & $0.47 \%$ & & 0 & 1 \\
\hline 72. & & LA & 60 & $0.33 \%$ & & 0 & 1 \\
\hline 73. & & MA & 27 & $0.15 \%$ & & 0 & 1 \\
\hline 74. & & MD & 390 & $2.15 \%$ & & 0 & 1 \\
\hline 75. & & ME & 2 & $0.01 \%$ & & 0 & 1 \\
\hline 76. & & MI & 201 & $1.11 \%$ & & 0 & 1 \\
\hline 77. & & MN & 25 & $0.14 \%$ & & 0 & 1 \\
\hline 78. & & MO & 52 & $0.29 \%$ & & 0 & 1 \\
\hline 79. & & MS & 74 & $0.41 \%$ & & 0 & 1 \\
\hline 80. & & MT & 12 & $0.07 \%$ & & 0 & 1 \\
\hline 81. & & $\mathrm{NC}$ & 115 & $0.63 \%$ & & 0 & 1 \\
\hline
\end{tabular}


$82 . \quad$ ND

$83 . \quad$ NJ

84. NM

$85 . \quad$ NV

$86 . \quad \mathrm{NY}$

87.

88.

89.

90.

91.

92.

93.

94.

95.

96.

97.

98.

99.

100.

\begin{tabular}{rrrr}
2 & $0.01 \%$ & 0 & 1 \\
11 & $0.06 \%$ & 0 & 1 \\
57 & $0.31 \%$ & 0 & 1 \\
33 & $0.18 \%$ & 0 & 1 \\
9,608 & $52.93 \%$ & 0 & 1 \\
3,604 & $19.85 \%$ & 0 & 1 \\
26 & $0.14 \%$ & 0 & 1 \\
10 & $0.06 \%$ & 0 & 1 \\
767 & $4.23 \%$ & 0 & 1 \\
59 & $0.33 \%$ & 0 & 1 \\
13 & $0.07 \%$ & 0 & 1 \\
156 & $0.86 \%$ & 0 & 1 \\
472 & $2.6 \%$ & 0 & 1 \\
159 & $0.88 \%$ & 0 & 1 \\
95 & $0.52 \%$ & 0 & 1 \\
4 & $0.02 \%$ & 0 & 1 \\
182 & $1 \%$ & 0 & 1 \\
37 & $0.2 \%$ & 0 & 1 \\
1 & $0.01 \%$ & 0 & 1 \\
\hline
\end{tabular}




\section{Definitions of Variables}

Variables 41 to 44. Education level in the community is measured using data from the U.S. Census Bureau American Community Survey. It includes four variables representing proportions of populations with different educational levels, such as:

41. No high school diploma (edf_nohs_pc)

42. High school diploma (edf_hs_ps)

43. Some college (edf_college_pc), and

44. College degree (edf_collegedegree_pc)

45. Income in the community is measured using data from the U.S. Census Bureau American Community Survey, which was recoded into a dummy variable whether the median income in less than \$50,000 (income_less50k_bin).

Variables 46 to 52. Four regions are identified following the U.S. Postal service distinction and include North-East which comprised of 7 states: ME, VT, PA, NY, NJ, MA, CT; Midwest with 11 states: ND, SD, KS, MN, IA, MO, WI, IL, MI, IN, OH; South with 17 states: TX, OK, AR, LA, KY, TN, WV, MD, DE, DC, VA, NC, SC, GA, FL, AL, MS; and West with 11 states: MT, WY, ID, OR, CO, UT, NV, CA, NM, AK, HI. These regions are recoded into two sets of variables:

46. A categorical variable (region) coded as 0.) Northeast, 1.) Midwest, 2.) South, and 3.) West And 4 dummy variables:

47. Northeast (region_neast)

48. West (region_mwest)

49. South (region_south)

50. West (region_west)

Also, two sets of dummy variables identify regions of Appalachia in accordance with the distinction of the Appalachian Regional Commission (ARC):

51. A dummy variable indicating Appalachia region(region_app)

52. A dummy variable indicating Southern Appalachia region (region_sapp)

53. Rurality of the community was measured using the rural-urban distinction of the Health Resources and Services Administration (HRSA). It was saved as a dummy variable (ru_hrsa2) with two categories: 1 . Urban, and 0 . Rural 
Variables 54 to 100. State of residence is coded using two sets of variables:

54. A string variable (state_cd)

55 to 100. A set of a dummy variable, including AK, AL, AR, CA, CO, CT, DC, DE, FL, GA, HI, IA, ID, IL, IN, KS, KY, LA, MA, MD, ME, MI, MN, MO, MS, MT, NC, ND, NJ, NM, NV, NY, OH, OK, OR, PA, SC, SD, TN, TX, UT, VA, WI, WV, and WY. 


\section{Clinical Characteristics}

Hospice Episodes ( $N=42,764)$

\begin{tabular}{|c|c|c|c|c|c|c|c|}
\hline$\#$ & Characteristic & Variable name & $\mathbf{n}$ & $\%$ & M(SD) & Min. & Max. \\
\hline 101. & Hospice LOS & total_los & - & - & $57.85(72.45)$ & 1 & 1099 \\
\hline 102. & Medical Team Size & coord_phy & - & - & $1.29(1.50)$ & 1 & 28 \\
\hline 103. & Hospice Live Discharge & fhospice_live_disch_episode & - & - & $20.39(6.69)$ & 0 & 216 \\
\hline 104. & Symptom management & sym_mgt_icd $\overline{9}$ & 9,482 & $22.17 \%$ & - & 0 & 1 \\
\hline 105. & 1 day Hospice & care_intensity_1day & 9,146 & $21.39 \%$ & - & 0 & 1 \\
\hline 106. & Hospice Transition ER & transition_er & 3,611 & $8.44 \%$ & - & 0 & 1 \\
\hline 107. & Primary Care Visits & physician_use & 14,418 & $33.72 \%$ & - & 0 & 1 \\
\hline 108. & Hospice Transition Inpatient & transition_inpt & 2,633 & $6.16 \%$ & - & 0 & 1 \\
\hline 109. & Coordination Insurance & coord_ins & 12,377 & $28.94 \%$ & - & 0 & 1 \\
\hline 110. & \# of episodes per child & ftotal_epi & - & - & $21.39(6.70)$ & 1 & 217 \\
\hline 111. & $\begin{array}{l}\text { Concurrent care (rx) } \\
\text { Costs }\end{array}$ & concurrent_care_rx & 9,725 & $22.74 \%$ & - & 0 & 1 \\
\hline 112. & Total costs & icosts_tot & - & - & $4,833(9,237)$ & 0 & 352,029 \\
\hline 113. & Fee-for-services & icosts_tot_ffs & - & - & $4,490(9228)$ & 0 & 352,029 \\
\hline 114. & Premium payment & icosts_tot_prem & - & - & $342(744)$ & 0 & 31,373 \\
\hline 115. & Inpatient & icosts_tot_ip & - & - & $1,555(5808)$ & 0 & 349,426 \\
\hline 116. & Outpatient & icosts_tot_ot & - & & $76(431)$ & 0 & 27,710 \\
\hline 117. & Prescription drugs & icosts_tot_rx & - & - & $455(1928)$ & 0 & 76,480 \\
\hline 118. & Labs & icosts_tot_lab & - & - & $59(254)$ & 0 & 9,442 \\
\hline 119. & Transportation & icosts_tot_transport & - & - & $16(113)$ & 0 & 10,000 \\
\hline 120. & Therapies & icosts tot therapies & - & - & $4(44)$ & 0 & 1,657 \\
\hline 121. & Hospice benefits & icosts_tot_hb & - & - & $619(1950)$ & 0 & 30,980 \\
\hline 122. & Registered nurse visits & icosts_tot_RN & - & - & $57(658)$ & 0 & 29,835 \\
\hline 123. & Durable medical equipment & icosts_tot_dme & - & - & $250(765)$ & 0 & 13,373 \\
\hline
\end{tabular}


Children $(\mathrm{N}=18,152)$

\begin{tabular}{|c|c|c|c|c|c|c|}
\hline Characteristic & Variable name & $\mathbf{n}$ & $\%$ & M(SD) & Min. & Max. \\
\hline 101. Hospice LOS & total_los & - & - & $57.85(72.45)$ & 1 & 1099 \\
\hline 102. Medical Team Size & coord phy & - & - & $1.29(1.50)$ & 1 & 28 \\
\hline 103. Hospice Live Discharge & fhospice_live_disch_episode & - & - & $20.39(6.69)$ & 0 & 216 \\
\hline 104. Symptom management & sym_mgt_icd 9 & 2,605 & $14.35 \%$ & - & & \\
\hline 105. 1day Hospice & care_intensity_1day & 9,146 & $50.39 \%$ & - & 0 & 1 \\
\hline 106. Hospice Transition ER & transition_er & 2,990 & $16.47 \%$ & - & 0 & 1 \\
\hline 107. Primary Care Visits & physician use & 9,657 & $53.20 \%$ & - & 0 & 1 \\
\hline 108. Hospice Transition Inpatient & transition_inpt & 1,806 & $9.95 \%$ & - & 0 & 1 \\
\hline 109. Coordination Insurance & coord_ins & 6,232 & $34.33 \%$ & - & 0 & 1 \\
\hline 110. \# of episodes per child & ftotal epi & - & - & $21.39(6.70)$ & 1 & 217 \\
\hline 111. Concurrent care & concurrent_care_rx & 62,43 & $34.39 \%$ & - & 0 & 1 \\
\hline Costs & & & & & & \\
\hline 112. Total costs & icosts tot & - & - & $2,566(8,374)$ & 0 & 352,029 \\
\hline 113. Fee-for-services & icosts_tot_ffs & - & - & $2,281(8,382)$ & 0 & 352,029 \\
\hline 114. Premium payment & icosts_tot_prem & - & - & $285(610)$ & 0 & 31,373 \\
\hline 115. Inpatient & icosts_tot_ip & - & - & $1,260(6,799)$ & 0 & 349,426 \\
\hline 116. Outpatient & icosts_tot_ot & - & - & $59(492)$ & 0 & 27,710 \\
\hline 117. Prescription drugs & icosts_tot_rx & - & - & $142(1062)$ & 0 & 76,480 \\
\hline 118. Labs & icosts_tot_lab & - & - & $43(252)$ & 0 & 9,442 \\
\hline 119. Transportation & icosts_tot_transport & - & - & $16(139)$ & 0 & 10,000 \\
\hline 120. Therapies & icosts_tot_therapies & - & - & $3(37)$ & 0 & 1,656 \\
\hline 121. Hospice benefits & icosts_tot_hb & - & - & $254(943)$ & 0 & 30,980 \\
\hline 122. Registered nurse visits & icosts_tot_RN & - & - & $38(578)$ & 0 & 29,834 \\
\hline 123. Durable medical equipment & icosts tot dme & - & - & $65(370)$ & 0 & 13,373 \\
\hline
\end{tabular}




\section{Definitions of Variables}

Variables 101 to 112. Clinical characteristics were computed based on episodes. For detailed information on how episodes were created see Appendix 1. All variables were created using MAX PS, OT, RX, and IP files.

101. Hospice LOS (total_los) is a continuous measure of the total length of all episodes in days.

102. Medical Team Size (coord phy) is a continuous measure of the number of providers who provided services during a hospice episode and had respective National Provider Identifier numbers (NPIs).

103. Hospice live discharge (fhospice_live_disch_episode) is a dummy indicator of hospital admission within after 2 days of hospice discharge.

104. Symptom management (sym_mgt_icd9) is an indicator of any services for constipation received during hospice episode. Constipation is defined using Rx_NDC codes 0904-7889; 0121-1154-00; 45802-868; 49348-189; 21130-693-38; ICD-9 codes: 564.0, 560.32, 787.61, $306.40,87.79,88.19$; and procedure codes 45915, 99511, 74000, 74010, and 74020.

105. 1-day hospice variable (care_intensity_1day) is a dummy indicator of a 1-day hospice episode.

106. The hospice transition ER variable (transition_er) is an indicator of the use of the ER office during hospice enrollment. ER use is defined by codes 99281; 99282; 99283; 99284; 99285.

107. The primary care visits vriable (physician_use) is an indicator of the use of physician's office during hospice enrollment. Physician's use is defined by OT procedure codes 99205 ; 99215; 99211; 99212; 99213; 99214; 99215; 99201; 99202; 99203; 99204.

108. Hospice transition inpatient (transition_inpt) is a dummy indicator of transition to inpatient services

109. Coordination insurance (coord_ins) is a dummy variable indicating whether a patient had private health insurance coverage for the each of 12 months of the year (el_pvt_ins_cd_1 to el_pvt_ins_cd_12)

110. The number of episodes per child (ftotal_epi) is defined as the total number of episodes per child.

125. The concurrent care (concurrent_care_rx) is a dummy vindicator of receiving concurrent hospice care services. It is defined as receiving any medication during a hospice episode. 
Variables 113 to 124. Costs of care were created using data from the MAX PS file. Each variable is calculated using a proportion where the numerator is the appropriate cost measure and the denominator is the total number of months the patient is eligible for Medicaid during the calendar year (el_elgblty_mo_cnt).

113. For the total costs variable (icosts_tot) the numerator is the total amount of money paid by Medicaid for the recipient during the calendar year for all types of service and any type of claim (tot_mdcd_pymt_amt).

114. For the fee-for-services cost variable (icosts_tot_ffs) the numerator is the amount of money paid by Medicaid for the recipient during the calendar year (tot_mded_ffs_pymt_amt) for the following types of services ( $\underline{\mathrm{TOS}})$ :

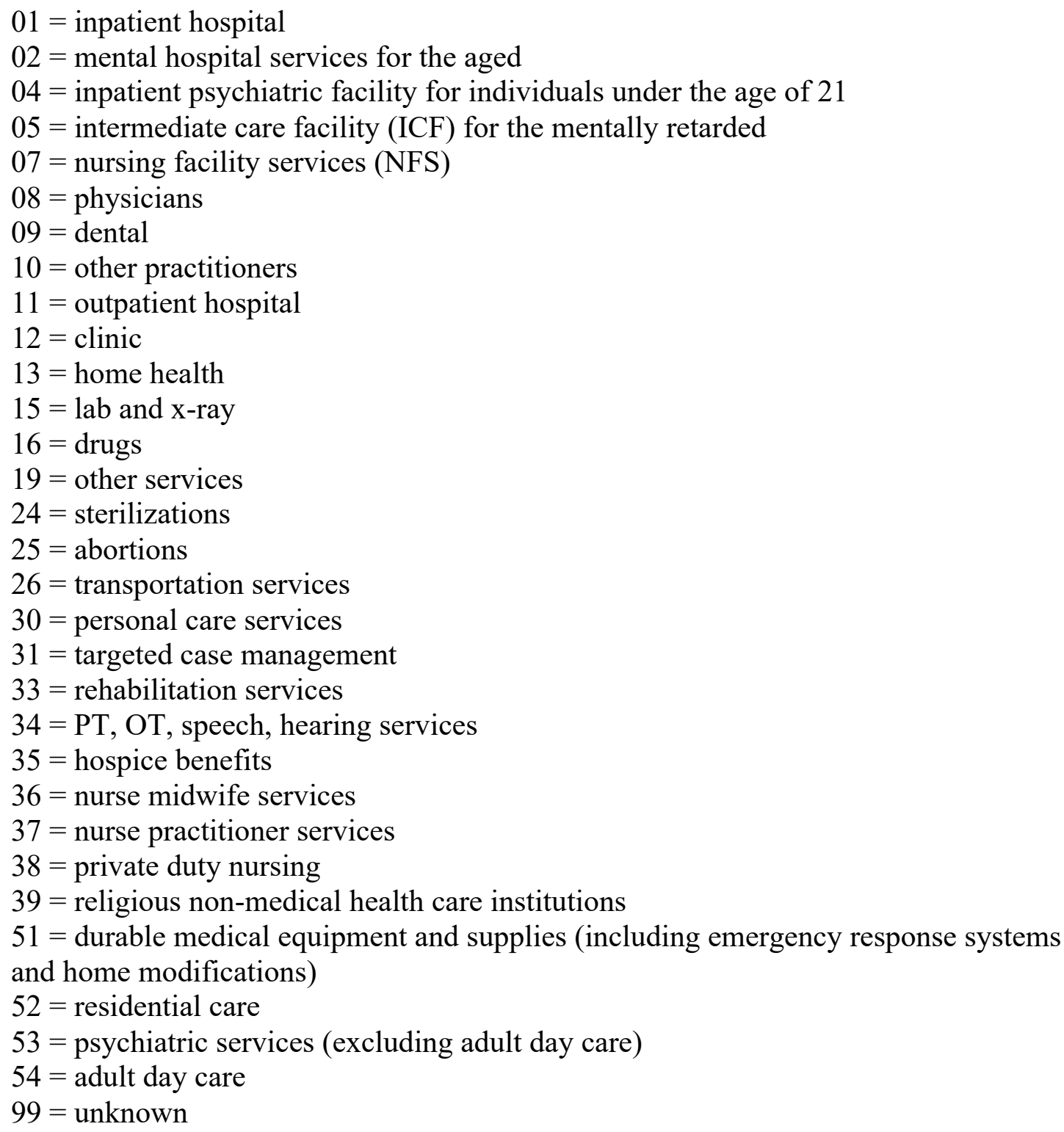


115. For the premium payment cost variable (icosts_tot prem) the numerator is the amount of money paid by Medicaid for the recipient during the calendar year (tot_mdcd_prem_pymt_amt), for the following TOS:

20 = Capitated payments to HMO, HIO, or PACE plans

21 = Capitated payments to prepaid health plans - PHPS

22 = Capitated payments for primary care case management - PCCM

116. For the inpatient cost (icosts_tot_ip) the numerator is the amount of money paid by Medicaid for the recipient during the calendar year for the inpatient hospital services (ffs_payment_amt_01).

117. For the outpatient cost (icosts_tot_ot) the numerator is the amount of money paid by Medicaid for the recipient during the calendar year for the outpatient hospital services (ffs_payment_amt_11).

118. For the prescription drugs cost (icosts_tot_rx) the numerator is the amount of money paid by Medicaid for the recipient during the calendar year for the drug prescriptions

(ffs_payment_amt_16).

119. For the labs cost (icosts_tot_lab) the numerator is the amount of money paid by Medicaid for the recipient during the calendar year for the lab and x-ray services (ffs_payment_amt_15).

120. For the transportation cost (icosts_tot_transport) the numerator is the amount of money paid by Medicaid for the recipient during the calendar year for the transportation services (ffs_payment_amt_26).

121. For the therapies cost (icosts_tot_therapies) the numerator is the amount of money paid by Medicaid for the recipient during the calendar year for the PT, OT, speech, hearing services (ffs_payment_amt_34).

122. For the Hospice benefits (icosts_tot_hb) the numerator is the amount of money paid by Medicaid for the recipient during the calendar year for the hospice benefits (ffs_payment_amt_35).

123. For the registered nurse visits cost (icosts_tot_rn) the numerator is the amount of money paid by Medicaid for the recipient during the calendar year for the nurse practitioner (ffs_payment_amt_37) and private duty nursing services (ffs_payment_amt_38).

124. For the durable medical equipment cost (icosts_tot_dme) the numerator is the amount of money paid by Medicaid for the recipient during the calendar year, for the durable medical equipment and supplies, including emergency response systems and home modifications (ffs_payment_amt_51). 


\section{Other Variables}

\begin{tabular}{|c|c|c|c|c|c|c|}
\hline \# & Characteristic & Variable name & n & $0 / 0$ & M(SD) & Min \\
\hline 124. & Medicaid waiver & waiver & 34,833 & $81.45 \%$ & 0 & 1 \\
\hline 125. & Palliative policy & instrument pall policy & 9,532 & $22.29 \%$ & 0 & 1 \\
\hline 126. & Hospice's postal ZIP code & - & - & - & - & - \\
\hline 127. & $\begin{array}{l}\text { Patient's postal ZIP code } \\
\text { Years }\end{array}$ & - & - & - & - & - \\
\hline 128. & 2011 & y2011 & 10,121 & $23.67 \%$ & 0 & 1 \\
\hline 129. & 2012 & y2012 & 7,472 & $17.47 \%$ & 0 & 1 \\
\hline 130. & 2013 & y2013 & 25,171 & $58.86 \%$ & 0 & 1 \\
\hline
\end{tabular}


Children $(\mathrm{N}=18,152)$

\begin{tabular}{|c|c|c|c|c|c|c|}
\hline \# & Characteristic & Variable name & $\mathbf{n}$ & $\%$ & M(SD) & Min. \\
\hline 124. & Medicaid waiver & waiver & 13,215 & $72.80 \%$ & 0 & 1 \\
\hline 125. & Palliative policy & instrument_pall_policy & 1,230 & $6.78 \%$ & 0 & 1 \\
\hline 126. & Hospice's postal ZIP code & - & - & - & - & - \\
\hline 127. & $\begin{array}{l}\text { Patient's postal ZIP code } \\
\text { Years }\end{array}$ & - & - & - & - & - \\
\hline 128. & 2011 & y2011 & 5,328 & $29.35 \%$ & 0 & ( \\
\hline 129. & 2012 & у2012 & 2,111 & 11.63 & 0 & 1 \\
\hline 130. & 2013 & у2013 & 11,218 & 61.80 & 0 & 1 \\
\hline
\end{tabular}




\section{Definitions of Variables}

Other variables were created using MAX PS files.

124. Medicaid waiver (waiver) is a dummy indicator of patient waiver enrollment for each of 12 months of the year (max_waiver_type_1_mo_1 to max_waiver_type_3_mo_12).

125. Instrument pall policy (instrument pall policy) is a dummy indicator of States with palliative policies, including California, Colorado, Illinois, Florida, Massachusetts, and Washington.

126. The hospices postal ZIP code (ziphosp) is a string variable that includes ZIP codes from the CMS PUF file.

127. The patient's postal ZIP code variable (ZIP) is a string variable that includes ZIP codes from the PS MAX file.

Variables 128-130. The year of data collection is coded into three dummy variables: y2011, y2012, and y2013. 


\section{Annotated List of Publications}

Cozad, M., Lindley, L. C., Eaker, C., Carlosh, K. A., \& Profant, T. L. (2019). Debunking myths about health insurance claims data for public health research and practice. American Journal of Public Health, 109(11), 1584-1585. https://doi.org/10.2105/AJPH.2019.305317

This paper examined three prevailing myths regarding claims data to raise awareness about their use in creating reliable evidence that supports public health practice.

Keim-Malpass, J., Cozad, M. J., Svynarenko, R., Mack, J. W., \& Lindley, L. C. (2021). Medical complexity and concurrent hospice care: A national study of Medicaid children from 2011 to 2013. Journal for Specialists in Pediatric Nursing, e12333. https://doi.org/10.1111/jspn. 12333 The objectives of this study were to examine the prevalence of concurrent hospice care over time and investigate the relationship between medical complexity and concurrent hospice care among Medicaid children.

Laird, J., Cozad, M. J., Keim-Malpass, J., Mack, J. W., \& Lindley, L. C. (2020). Variation in state Medicaid implementation of the ACA: The case of concurrent care for children. Health Affairs, 39(10), 1770-1775. https://doi.org/10.1377/hlthaff.2020.01192

This paper examined the state-level implementation of concurrent care for Medicaid beneficiaries and found significant variability in guidelines across the US.

Lindley, L. C., Cozad, M. J., Mack, J. W., Keim-Malpass, J., Svynarenko, R., \& Hinds, P. S. (2021). Effectiveness of pediatric concurrent hospice care to improve continuity of care. The American Journal of Hospice \& Palliative Care, 10499091211056040

https://doi.org/10.1177/10499091211056039

The purpose of this study was to test the hypotheses that children who used concurrent hospice care would have a longer length of stay in hospice, reduced disenrollment from hospice, and fewer transitions to the emergency room or inpatient setting, compared to those using standard hospice care.

Lindley, L. C., Cozad, M. J., Svynarenko, R., Keim-Malpass, J., \& Mack, J. W. (2021). A national profile of children receiving pediatric concurrent hospice care, 2011 to 2013. Journal of Hospice and Palliative Nursing, 23(3).

This study conducted a descriptive analysis of the demographic, community, hospice, and clinical characteristics of children who received concurrent hospice care between 2011 and 2013.

Lindley, L. C., Cozad, M. J., \& Fortney, C. A. (2020). Pediatric complex chronic conditions: Evaluating two versions of the classification system. Western Journal of Nursing Research, 42(6), 454-461. https://doi.org/10.1177/0193945919867266 The purpose of this study was to generate data on the complex chronic conditions (CCC) classification applied to infants by examining the prevalence of CCCs and investigating the characteristics related to a CCC classification in a national sample of infants less than 1 year. 
Lindley, L. C., \& Fortney, C. A. (2019). Pediatric complex chronic conditions: Does the classification system work for infants? American Journal of Hospice and Palliative Medicine ${ }^{\circledR}$, 104990911983898. https://doi.org/10.1177/1049909119838985

This research examined the prevalence of complex chronic conditions (CCC) and the infant characteristics related to a $\mathrm{CCC}$ classification

Lindley, L. C., Fortney, C. A., \& Cozad, M. J. (2021). Predictive ability of an illness severity measure: Implications for nursing research. Journal of Nursing

Measurement. https://doi.org/10.1891/JNM-D-19-00106

The objective of this study was to compare the predictive performance of the original (Feudtner et al., 2000; Feudtner et al., 2001) versus the revised (Feudtner et al., 2014) complex chronic conditions (CCC) measure among infants.

Lindley, L. C., Keim-Malpass, J., Cozad, M. J., Mack, J. W., Svynarenko, R., Fornehed, M. L. C., Stone, W., Qualls, K., \& Hinds, P. S. (2022). A national study to compare effective management of constipation in children receiving concurrent versus standard hospice care. Journal of Hospice and Palliative Nursing: JHPN: The Official Journal of the Hospice and Palliative Nurses Association, 24(1), 70-77. https://doi.org/10.1097/NJH.0000000000000810 The purpose of this study was to evaluate the effectiveness of pediatric concurrent hospice versus standard hospice care to manage constipation.

Lindley, L. C., Keim-Malpass, J., Svynarenko, R., Cozad, M. J., Mack, J. W., \& Hinds, P. S. (2020). Pediatric concurrent hospice care: A scoping review and directions for future nursing research. Journal of Hospice \& Palliative Nursing, 22(3), 238-

245. https://doi.org/10.1097/NJH.0000000000000648

The purpose of this study was to systematically collect the evidence on concurrent hospice care, critically appraise the evidence, and identify areas for future nursing research.

Lindley, L. C., Svynarenko, R., \& Beebe, L. H. (2021). Mental health and developmental disabilities in US children admitted in hospice care. International Journal of Palliative Nursing, 27(3), 124-130. https://doi.org/10.12968/ijpn.2021.27.3.124

This paper aimed to describe the mental health and developmental disabilities of children who were admitted to hospice care and compare them across age groups.

Lindley, L. C., Svynarenko, R., Mooney-Doyle, K., Mendola, A., Naumann, W. C., \& Fortney, C. A. (2021a). End-of-life healthcare service needs among children with neurological conditions: A latent class analysis. The Journal of Neuroscience Nursing: Journal of the American Association of Neuroscience Nurses. https://doi.org/10.1097/JNN.0000000000000615

This paper had 2 aims: to apply latent class analysis to identify patterns of nonhospice, healthcare service needs among children with neuro- logical conditions; and to assess the characteristics of latent class membership.

Lindley, L. C., Svynarenko, R., Mooney-Doyle, K., Mendola, A., Naumann, W., \& KeimMalpass, J. (2021b). Patterns of health care services during pediatric concurrent hospice care: A national study. American Journal of Hospice \& Palliative Medicine, 1-7. https://doi.org/10.1177/10499091211018661 
The purpose of this study was to examine the health care services received by children enrolled in concurrent hospice care, unique clusters of health care services, and characteristics of the children in the clusters.

Lindley, L. C., Svynarenko, R., \& Profant, T. L. (2020). Data infrastructure for sensitive data: Nursing's role in the development of a secure research enclave. Computers, Informatics, Nursing: CIN, 38(9), 427-430. https://doi.org/10.1097/CIN.0000000000000677

This paper describes the development of a secure data infrastructure designed to work with protected health information (PHI), personally identifiable information (PII), or sensitive data.

Mendola, A., Naumann, W. C., Mooney-Doyle, K., \& Lindley, L. C. (2021). Social determinants of comfort: A new term for end-of-life care. Journal of Palliative Medicine, 24(8), 1130-

1131. https://doi.org/10.1089/jpm.2021.0209

This study proposed the term social determinants of comfort to describe structural conditions that influence whether, to what degree, and in what forms comfort measures are offered to and accepted by patients and their families.

Mooney-Doyle, K., Keim-Malpass, J., Svynarenko, R., \& Lindley, L. C. (2021). A comparison of young adults with and without cancer in concurrent hospice care: Implications for transitioning to adult health care. Journal of Adolescent and Young Adult Oncology. https://doi.org/10.1089/jayao.2021.0004

This study compared young adults with and without cancer in concurrent hospice care.

Stone, W., Keim-Malpass, J., Cozad, M. J., Fornehed, M. L. C., \& Lindley, L. C. (2021). Pediatric end-of-life care in rural America: A systematic review. The American Journal of Hospice \& Palliative Care, 10499091211064202. https://doi.org/10.1177/10499091211064202 The purpose of this study was to comprehensively review and summarize the evidence regarding end-of-life care for children living in rural areas, identify key findings and gaps in the literature, and make recommendations for future research.

Svynarenko, R., Beebe, L. H., \& Lindley, L. C. (2021). Identifying patterns of pediatric mental and behavioral health at end of life: A national study. Journal of Hospice \& Palliative Nursing, Publish Ahead of Print. https://doi.org/10.1097/NJH.0000000000000800 the purpose of this study was to identify patterns of mental and behavioral health of children enrolled in hospice care and describe the demographic and health characteristics of children in these groups.

Svynarenko, R., \& Lindley, L. (2021). Defining rurality in end-of-life research: Evaluation of common measures. The Journal of Health Care for the Poor and Underserved, 32(4), 21672180. https://doi.org/10.1353/hpu.2021.0189

The purpose of this study was to conduct a comprehensive evaluation of eight rural-urban classifications against the gold standard of the Office of Budget and Management to determine the utility of alternative measures in hospice research. 
Svynarenko, R., Mack, J. W., \& Lindley, L. C. (2021). Differences in characteristics of children with cancer who receive standard versus concurrent hospice care. Pediatric Blood \& Cancer. https://doi.org/10.1002/pbc.29106

The purpose of the study was to compare demographic, health, and community characteristics of children who received standard hospice care versus concurrent hospice care.

Lindley, Lisa C., Radion Svynarenko, Kim Mooney-Doyle, Annette Mendola, Wendy C. Naumann, and Robin Harris. "A National Study of Healthcare Service Patterns at the End of Life among Children with Cardiac Disease." The Journal of Cardiovascular Nursing, December 21, 2021. https://doi.org/10.1097/JCN.0000000000000875.

The aims of this study were to identify patterns of nonhospice healthcare services used in concurrent hospice care and describe the profile of children with cardiac disease in these clusters. 


\section{Appendix: Creating Hospice Episodes}

First, our team presents rules for episodes and then how to make episodes using manual transformation and syntax.

\section{Rules for hospice episodes:}

1. A hospice episode is a period of time when a patient receives hospice services.

2. There are two types of episodes: (a) single-day episodes and (b) multiple-day episodes with subsequent days of service.

3. An episode begins on the first day of service and ends when consecutive days of services come to end.

4. For single-day episodes, the first and the last day are the same days.

5. If there is a break between services for more than one day, these services belong to different episodes.

6. Episodes do not have duplicate dates.

7. The same person may have multiple episodes.

\section{Manual transformations}

We have three people (\#6, \#7, \#8) with nine service days. They are sorted by ID and DATE.

\begin{tabular}{rl}
\hline ID & Date \\
\hline 6 & 20120205 \\
6 & 20120206 \\
6 & 20120207 \\
7 & 20111209 \\
7 & 20120101 \\
7 & 20120102 \\
8 & 20110103 \\
8 & 20110103 \\
8 & 20110104 \\
\hline
\end{tabular}

We can identify four episodes:

$1,2,3,4$.

Episode \#4 for person \#8 has one duplicate record: 20110103. It must be eliminated (rule \#5)

\begin{tabular}{rlr}
\hline ID & Date & Episode \# \\
\hline 6 & 20120205 & 1 \\
6 & 20120206 & 1 \\
6 & 20120207 & 1 \\
7 & 20111209 & 2 \\
7 & 20120101 & 3 \\
7 & 20120102 & 3 \\
8 & 20110103 & 4 \\
8 & 20110103 & 4 \\
8 & 20110104 & 4 \\
\hline
\end{tabular}

Then, we can reshape it wide based on episode \#.

\begin{tabular}{lllll}
\hline Episode \# & ID & Start & End & LOS, days \\
\hline 1 & 6 & 20120205 & 20120207 & 4 \\
2 & 7 & 20111209 & 20111209 & 1 \\
3 & 7 & 20120101 & 20120102 & 2 \\
4 & 8 & 20110103 & 20110104 & 1 \\
\hline
\end{tabular}

Transformations with $\operatorname{syntax}^{1}$ :

${ }^{1}$ Syntax is borrowed from STATA's forum https://www.statalist.org/forums/forum/generalstata-discussion/general/1415997-identifying-spells More example on episodes management are in STATA's stset manual https://www.stata.com/manuals13/ststset.pdf 
First: generate STATA readable dates, variable date2

. gen date2= date (date, "YMD")

. format $\%$ td date 2

. drop date

\begin{tabular}{rll}
\hline ID & date & date2 \\
\hline 6 & 20120205 & 05feb2012 \\
6 & 20120206 & 06feb2012 \\
6 & 20120207 & 07feb2012 \\
7 & 20111209 & 09dec2011 \\
7 & 20120101 & 01jan2012 \\
7 & 20120102 & 02jan2012 \\
8 & 20110103 & 03jan2011 \\
8 & 20110103 & 03jan2011 \\
8 & 20110104 & 05jan2011 \\
\hline
\end{tabular}

Second: sort by id and date2, and then identify gaps between observations within each ID.

. sort id date2

/*sorting*/

. by id: gen gap $=$ date2 - date2[ $\mathrm{n}-1]$

/*gaps between observations within each ID*/

How it works:

The third $(\mathrm{n}=3$ ) record in id\#6 is 07 feb2012 minus the previous record ( $\mathrm{n}$ -

1) is $06 \mathrm{feb} 2012$, and the difference between them is equal to a 1-day gap.

Eighth $(\mathrm{n}=8)$ record in id\#8 is 03jan2011 minus previous record $(\mathrm{n}-1)$ is

03jan2011 and equal to a 0-day gap, a duplicate record. Lines 1,4 and 7 are missing because they are the first ones within each new ID

\begin{tabular}{rlll}
\hline ID & date & Date 2 & gap \\
\hline 6 & $05 f$ feb2012 &. \\
6 & $06 f$ feb2012 & 1 \\
6 & 07 feb2012 & 1 \\
7 & 09 dec2011 &. \\
7 & 01 jan2012 & 23 \\
7 & 02 jan2012 & 1 \\
8 & 03 jan2011 &. \\
8 & 03jan2011 & 0 \\
8 & 04 jan2011 & 1 \\
\hline
\end{tabular}

*Third: identify uninterrupted episodes (i.e., spells) within each ID.

Interrupted episodes do not have time gaps of more than one day (see rule\#4). In other words, if a gap is longer than one day, a new episode must be started.

.by id: gen spell $=$ sum $($ gap $>1)$

Don't get confused. Mathematically, the gap between 06feb2012 and 07 feb2012 is equal to 1 . Because $07-06=1$. But we understand that it is just the next day's event, and both days belong to the same spell. This is why the syntax says gap $>1$; in syntax, the gap should be at least two days to start a new spell.

\begin{tabular}{llcc}
\hline ID & date2 & gap & spell \\
\hline 6 & $05 f$ feb2012 &. & 1 \\
6 & $06 f$ feb2012 & 1 & 1 \\
6 & $07 f e b 2012$ & 1 & 1 \\
7 & 09 dec2011 &. & 1 \\
7 & 01 jan2012 & 23 & 2 \\
7 & 02 jan2012 & 1 & 2 \\
8 & 03jan2011 &. & 1 \\
8 & 03jan2011 & 0 & 1 \\
8 & $04 j a n 2011$ & 1 & 1 \\
\hline
\end{tabular}

Fourth: grouping observations by spell and id, in other words ordernumbering all spells .egen spellid $=$ group $(\mathrm{id}$ spell $)$

\begin{tabular}{llccc}
\hline ID & date2 & gap & spell & spellid \\
\hline 6 & 05feb2012 &. & 1 & 1 \\
6 & 06feb2012 & 1 & 1 & 1 \\
6 & $07 \mathrm{feb2012}$ & 1 & 1 & 1 \\
7 & 09 dec2011 &. & 1 & 2 \\
7 & 01 jan2012 & 23 & 2 & 3 \\
7 & 02jan2012 & 1 & 2 & 3 \\
8 & 03jan2011 &. & 1 & 4 \\
8 & 03jan2011 & 0 & 1 & 4 \\
8 & 04jan2011 & 1 & 1 & 4 \\
\hline
\end{tabular}


Fifth: identify start- and end- dates, and also length of service

.bysort spellid (date2): egen start $=\min ($ date2)

/*start date is the minimum value in the column date2 within each ID*/

. bysort spellid (date2): egen end $=\max ($ date 2$)$

$1 *$ end date is the maximum value in the column date2 within each ID*/

.format start end \%td $/ *$ making sure it is in stata's time format*/

.gen length $=$ end-start +1

\begin{tabular}{llllllll}
\hline ID & date2 & gap & spell & spellid & start & end & LOS \\
\hline 6 & 05feb2012 &. & 1 & 1 & 05feb2012 & 07feb2012 & 3 \\
6 & $06 f e b 2012$ & 1 & 1 & 1 & 05feb2012 & 07feb2012 & 3 \\
6 & $07 f e b 2012$ & 1 & 1 & 1 & 05feb2012 & 07feb2012 & 3 \\
7 & 09dec2011 &. & 1 & 2 & 09dec2011 & 09dec2011 & 1 \\
7 & 01 jan2012 & 23 & 2 & 3 & 01jan2012 & 02jan2012 & 2 \\
7 & 02jan2012 & 1 & 2 & 3 & 01jan2012 & 02jan2012 & 2 \\
8 & 03jan2011 &. & 1 & 4 & 03jan2011 & 04jan2011 & 2 \\
8 & 03jan2011 & 0 & 1 & 4 & 03jan2011 & 04jan2011 & 2 \\
8 & 04jan2011 & 1 & 1 & 4 & 03jan2011 & 04jan2011 & 2 \\
\hline
\end{tabular}

Sixth: dropping duplicates. We could separately drop duplicate record for 03jan2011 (rule \#5). But because we have to drop duplicates anyways, one command will do both jobs. This is the last line.

.duplicates drop spellid start end, force

\begin{tabular}{|c|c|c|c|c|c|c|c|}
\hline ID & date2 & gap & spell & spellid & start & end & LOS \\
\hline 6 & 05feb2012 & . & 1 & 1 & 05feb2012 & 07feb2012 & 3 \\
\hline 6 & 06feb2012 & 4 & 4 & 4 & 05 feb2012 & $07 \mathrm{feb} 2012$ & 3 \\
\hline 6 & $07 \mathrm{feb} 2012$ & 4 & 4 & 4 & 05 feb 2012 & $07 \mathrm{feb} 2012$ & 3 \\
\hline 7 & 09dec2011 & . & 1 & 2 & 09dec2011 & 09dec2011 & 1 \\
\hline 7 & 01 jan2012 & 23 & 2 & 3 & $01 \mathrm{jan} 2012$ & 02jan2012 & 2 \\
\hline 7 & $02 \mathrm{jan} 2012$ & 4 & 2 & 3 & 01 jan2012 & $02 \mathrm{jan} 2012$ & 2 \\
\hline 8 & 03jan2011 & . & 1 & 4 & 03jan2011 & 04jan2011 & 2 \\
\hline 8 & 03 jan2011 & $\theta$ & 4 & 4 & 03 jan2011 & 04 jan2011 & $z$ \\
\hline 8 & 04jan2011 & 4 & 4 & 4 & 03 jan2011 & 04 jan2011 & $z$ \\
\hline
\end{tabular}




\begin{tabular}{|c|c|c|c|c|c|c|c|}
\hline \multicolumn{8}{|c|}{ Merging databases } \\
\hline $\begin{array}{l}\text { Episode } \\
\#\end{array}$ & Msis_ID & Start & End & Ot & npi & procedure & date \\
\hline \multirow[t]{5}{*}{1} & \multirow[t]{5}{*}{6} & \multirow[t]{5}{*}{20120205} & \multirow[t]{5}{*}{20120207} & & . & & 20120207 \\
\hline & & & & 1 & 1 & & 20120207 \\
\hline & & & & 1 & 3 & & 20120207 \\
\hline & & & & 4 & 4 & & 20120207 \\
\hline & & & & 1 & 3 & & 20120207 \\
\hline \multirow[t]{6}{*}{2} & \multirow[t]{6}{*}{7} & \multirow[t]{6}{*}{20111209} & \multirow[t]{6}{*}{20111209} & & & & 20111209 \\
\hline & & & & 1 & 4 & & 20111209 \\
\hline & & & & 1 & 5 & & 20111209 \\
\hline & & & & 4 & 6 & & 20111209 \\
\hline & & & & 1 & 4 & & 20111209 \\
\hline & & & & 1 & 5 & & 20111209 \\
\hline \multirow[t]{5}{*}{3} & \multirow[t]{5}{*}{7} & \multirow[t]{5}{*}{20120101} & \multirow[t]{5}{*}{20120102} & . & & & 20120102 \\
\hline & & & & 1 & 7 & & 20120102 \\
\hline & & & & 4 & 8 & & 20120102 \\
\hline & & & & 1 & 9 & & 20120102 \\
\hline & & & & 1 & 8 & & 20120102 \\
\hline \multirow[t]{6}{*}{4} & \multirow[t]{6}{*}{8} & \multirow[t]{6}{*}{20110103} & \multirow[t]{6}{*}{20110104} & & & & 20110104 \\
\hline & & & & 1 & 9 & & 20110104 \\
\hline & & & & 4 & 10 & & 20110104 \\
\hline & & & & 1 & 10 & & 20110104 \\
\hline & & & & 1 & 9 & & 20110104 \\
\hline & & & & 1 & 9 & & 20110104 \\
\hline
\end{tabular}

* creating database with services

1. create a database with procedures, which has two variables: msis_id and date.

* working with episodes

**removing cases that do not fit within the episode's time frame

2. append procedures with episodes

3. refill empty dates with the START date

4. sort by msis_id and date

5. expand episode's id down to all cases within the person

6. drop episode's id that have missing values

7. within episode's id remove cases with the date that is later than the END date

8. remove cases that have dates later than the END date

** counting NPIs

9. sort by episodes and NPIs

10. Remove duplicate NPI within each episode

11. count total number of cases within each episode. 